\title{
O Efeito Casimir para um Campo Fermiônico na presença de um Campo Magnético Externo
}

\author{
The Casimir Effect of a Fermionic Field in the Presence of \\ an External Magnetic Field
}

\author{
M.S.R. Miltão* e Franz A. Farias ${ }^{\dagger}$ \\ Departamento de Física - UEFS \\ Campus Universitário, Km 03 BR 116 \\ Feira de Santana - BA - 44031-460
}

\begin{abstract}
Neste trabalho revisitamos a influência de um campo externo uniforme, particularmente de um campo magnético, sobre a energia de Casimir de um campo fermiônico [1, 2]. Recalculamos então o efeito Casimir nas condições citadas utilizando uma forma generalizada para as condições de contorno sobre o campo bem como o método da função zeta generalizada [3], o qual permite extrair um resultado final mais "limpo", i.e., um com menos termos divergentes espúrios.
\end{abstract}

Palavras-chaves: Efeito Casimir, Função Zeta Generalizada, Campo Magnético Externo, Campo de Dirac.

In this work we review of the influence of an external constant field, particularly a magnetic one, on the Casimir energy of a fermionic field $[1,2]$. We recalculate then the Casimir effect under the conditions using a generalized form for the boundary conditions imposed on the field as well as the method of generalized zeta function [3]. The latter allows extracting "cleaner" result, i.e., one with less spurious divergent terms.

Key-words: Casimir Effect, Generalized Zeta Function, External Magnetic Field, Dirac Field.

\section{INTRODUÇÃO}

O efeito Casimir tem sido um tema cujo interesse tem se mantido crescente desde seu surgimento em 1948 com o trabalho do físico holandês que dá seu nome [4]. Inicialmente considerado para o campo eletromagnético quantizado entre duas placas condutoras, seu estudo e aplicação estendeuse para outros campos quânticos a exemplo dos campos de calibre com simetria BRST, do campo de Higgs, dos campos com supersimetria, da teoria de supergravidade, das supercordas, do campo Maxwell-Chern-Simons, da corda relativística, da teoria $\mathrm{M}$, nos temas relacionados com cosmologia, nos relacionados com espaços não-comutativos, dentre outros.

De uma forma mais ampla o efeito Casimir, num sentido lato, pode ser definido [5] como os efeitos que decorrem da variação da energia de ponto zero de um campo quântico relativístico, devido às mudanças de sua variedade de base $\mathscr{M}$. Sendo assim, podemos realizar uma classificação livre do efeito Casimir num conjunto de situações como segue:

(i) quando a mudança na variedade de base ocorre devido a existência de condições de contorno - efeito Casimir devido a condições de contorno, que é o caso do efeito Casimir original [4], onde as placas condutoras mudam a variedade de base de $\mathbb{R}^{3}$ para $\mathbb{R}^{2} \times[0, a]$;

(ii) quando a mudança na variedade de base ocorre devido a existência de meios materiais - efeito Casimir em meios materiais $[6,7]$;

\footnotetext{
*Endereço Eletrônico: miltaaao@ig.com.br

†Endereço Eletrônico: franz_farias@terra.com.br
}

(iii) quando a mudança na variedade de base ocorre devido a existência de um campo gravitacional - efeito Casimir em espaços curvos [8];

(iv) quando a mudança na variedade de base ocorre devido a alterações nas propriedades algébricas das coordenadas do espaço-tempo ou quando a álgebra associada ao grupo de simetria do sistema físico em questão sofre uma deformação. No primeiro caso temos por exemplo que $[x, y]=x y-y x \neq 0$, ou seja, as coordenadas tornam-se operadores [9], enquanto que no segundo caso existe um parâmetro $\theta$ ou $\kappa$ [10] que deforma a álgebra de Poincaré usual [11]. Em ambos os casos temos o efeito Casimir em estruturas matemáticas deformadas.

O interesse no efeito Casimir reside essencialmente no fato de que todo campo quântico possui uma energia de ponto zero, i.e., em seu estado fundamental (estado de vácuo) todo campo quântico relativístico tem uma energia dada por

$$
\mathcal{E}_{0}=\sum_{n} \frac{1}{2} \hbar \omega_{n}
$$

onde $n$ enumera os modos normais de oscilação do campo em questão, $\omega_{n}$ é a freqüência do n-ésimo modo, e a soma inclui todos os modos normais.

Casimir [4] previu que duas placas condutoras paralelas, bem próximas e descarregadas, alterariam a energia do vácuo quântico eletromagnético por uma quantidade $\mathcal{E}(a)$ dada por

$$
\frac{\mathcal{E}(a)}{\ell^{2}}=-\frac{\pi^{2}}{720 a^{3}} \hbar c,
$$

onde $\ell^{2}=L_{x} L_{y}$ é a área de cada uma das placas, supostas quadradas por simplicidade, e $a$ é a separação entre 
elas $(a \ll \ell)$ (condição de pequena separação), conforme a Figura 1 abaixo.

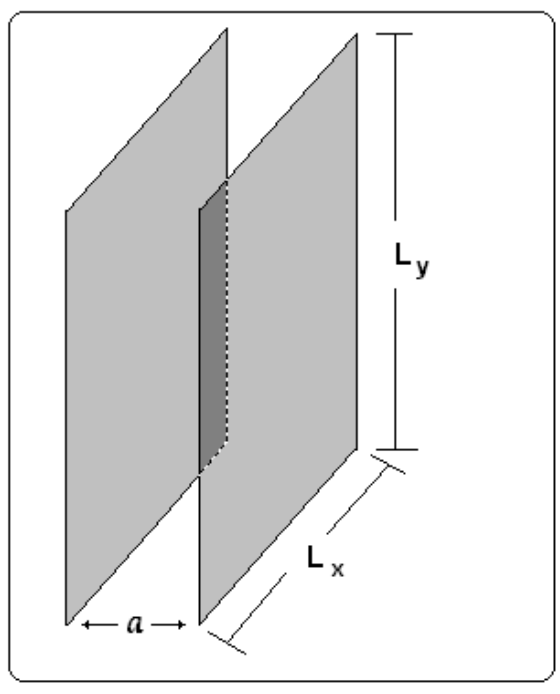

Fig. 1 - O efeito Casimir original

A atração entre corpos neutros não era novidade quando Casimir propôs o efeito que leva o seu nome. De fato, essa atração era conhecida e explicada como efeito das forças de van der Waals de dispersão, isto é, forças eletromagnéticas oriundas da interação entre dipolos induzidos mutuamente, em virtude de flutuações quânticas, em átomos que são neutros e despolarizados quando isolados. A originalidade de Casimir consistiu, por um lado, em atribuir a força de atração entre os corpos neutros à alteração que eles provocam na energia de ponto zero do vácuo eletromagnético e, por outro lado, em usar essa idéia para obter a força de atração pelo cálculo da dita alteração, um método incomparavelmente mais simples do que calcular superposições de forças de van der Waals entre moléculas.

Por sua vez, a energia $\mathcal{E}(a)$ dá origem à seguinte pressão entre as placas

$$
P(a)=\frac{F(a)}{\ell^{2}}=-\frac{\pi^{2}}{240 a^{4}} \hbar c=-0,013 \frac{1}{(a / \mu m)^{4}} \frac{d y n}{c m^{2}},
$$

onde o sinal negativo significa que a pressão é atrativa. Esse efeito de atração é o efeito Casimir original. Ele foi confirmado experimentalmente por Sparnaay em 1958 (apesar da grande imprecisão experimental) [12] e, mais recentemente com grande acurácia (em torno de 1\%), por Lamoreaux [13] e por Mohideen e Roy [14].

A energia de Casimir referida acima é devida a um campo quântico bosônico, no caso a do campo de radiação. Foi Johnson [15] quem calculou pela primeira vez a energia de Casimir de um campo de Dirac não-massivo, considerando a condição de fronteira do tipo sacola do M.I.T. na configuração de confinamento de duas placas paralelas separadas por uma distância $a$. A energia de Casimir encontrada por Johnson [15] foi:

$$
\frac{\mathcal{E}(a)}{\ell^{2}}=-\xi \frac{\pi^{2}}{720 a^{3}} \hbar c
$$

onde $\xi=7 / 4$. Como comentamos acima, o efeito Casimir surge de uma mudança da variedade de base e nesse caso do efeito Casimir fermiônico esta energia surge da mudança do espaço $\mathbb{R}^{3}$ para o espaço $\mathbb{R}^{2} \times[0, a]$. A motivação para o cálculo de Johnson foi entender, usando o efeito Casimir, o mecanismo de confinamento dos quarks nos hádrons. Claro está que um cálculo mais realístico para o citado confinamento deveria levar em conta a geometria esférica e este passo só foi realizado posteriormente por K. Milton [16] e mais recentemente por E. Elizalde [17], o qual considera um campo fermiônico massivo.

A idéia primária que se coloca neste trabalho é, antes de tudo, a de uma investigação das alterações que sofrem as flutuações de vácuo de um campo fermiônico carregado quando sobre o mesmo se impõem condições de contorno e também campo externos, em particular um magnético. Como mostrado em [2], onde o efeito Casimir fermiônico é calculado usando o método de Schwinger, e [18], onde o cálculo é realizado usando o método de soma de modos, a presença de um campo magnético uniforme externo ressalta o efeito Casimir, um resultado que também confirmaremos neste trabalho. Cabe frisar que este comportamento está em oposição àquele devido a campo bosônico, para o qual a energia de Casimir associada é inibida pela presença do campo externo, como mostrado em [19].

Como bem observaram M.V. Cougo-Pinto et al. [2], o resultado para a energia de Casimir de um campo fermiônico não-massivo sob as condições de contorno do tipo M.I.T., periódica ou antiperiódica apresentam a mesma dependência com a distância $a^{3}$, a exceção do valor do fator $\xi$ que no caso periódico vale $7 \times 4$ enquanto que no antiperiódico vale $-8 \times 4$. Isto significa que a mudança de base dada por $\mathbb{R}^{2} \times S^{1}$, que corresponde a uma compactificação de uma das dimensões espaciais num círculo, e a primeira situação acima referida, guardam semelhanças.

Sendo assim, adotaremos as condições de contorno periódica e antiperiódica para uma primeira avaliação da energia de Casimir do campo fermiônico. Na realidade, construiremos uma condição de contorno generalizada através de um parâmetro apropriado, como veremos mais a frente, de tal maneira que as condições de contorno periódica e antiperiódica surgem como casos particulares em valores definidos do parâmetro $\alpha$, precisamente $\alpha=0$ e $\alpha=\pi$ respectivamente.

Neste trabalho revisitamos o efeito Casimir de um campo fermiônico sob a influência de um campo magnético uniforme, generalizando a forma da condição de contorno antiperiódica imposta ao campo fermiônico em [2] através de um parâmetro apropriado. Calculamos o efeito Casimir fermiônico usando o método da função zeta generalizada para obter um resultado com um mínimo de termos divergentes espúrios e, por fim, analisamos o resultado e suas conseqüências comparando com aqueles conhecidos na literatura.

O artigo encontra-se organizado como segue. Na próxima seção apresentamos a idéia do método da função zeta generalizada, sua construção, extensão analítica e o cálculo de sua derivada no ponto $s=0$. Para tanto devemos resolver o traço do operador de Dirac, ou mais propriamente, do operador quadrático correspondente, e isto é conseguido nas $3^{\mathrm{a}}$ e $4^{\mathrm{a}}$ seções. O passo seguinte na seção 5 é a determinação do espectro do citado operador. A construção da função zeta ocorre na seção 6 enquanto que seu desenvolvimento é realizado nas seções 7 e 8 . Na $9^{a}$ seção realizamos uma análise do resultado obtido na seção anterior. Apresentamos então as conclusões. No apêndice encontra-se detalhado a obtenção do fator de degenerescência dos níveis de Landau. 


\section{O MÉTODO DA FUNÇÃO ZETA GENERALIZADA}

O ponto de partida é a densidade de lagrangeana do campo de Dirac:

$$
\mathcal{L}=-\bar{\psi}(\gamma \cdot \Pi+m) \psi
$$

onde $\Pi_{\mu}$ é dado por

$$
\Pi_{\mu}=\frac{1}{i} \partial_{\mu}-e A_{\mu}^{e}
$$

O campo magnético neste problema é um campo constante e uniforme, e sendo assim, pode ser construído a partir do seguinte potencial

$$
A_{\mu}^{e}(x)=(0,-B y, 0,0) .
$$

As equações para os campos $\psi$ e $\bar{\psi}$ que resultam da den- sidade de lagrangeana em (5) são

$$
(\gamma \cdot \Pi+m) \psi=0 \quad \text { e } \quad \bar{\psi}\left(\gamma \cdot \Pi^{*}+m\right)=0
$$

A densidade de energia do vácuo (energia por unidade de área) do campo fermiônico pode ser obtida do conhecimento da amplitude de persistência do vácuo.

A amplitude de persistência do vácuo na presença de um campo externo está relacionada ao funcional gerador das funções de Green conexas, $W$, [20]

$$
\begin{aligned}
& \left\langle 0_{+} \mid 0_{-}\right\rangle_{\alpha}^{\bar{\eta}, \eta}=\exp \left(i W_{\alpha}\left[A^{e}, \bar{\eta}, \eta\right]\right) \\
= & \int_{C_{\alpha}} \mathcal{D} \bar{\psi} \mathcal{D} \psi \exp \left\{i \int d^{4} x[\mathcal{L}(x)+\bar{\eta} \psi+\bar{\psi} \eta]\right\} .
\end{aligned}
$$

Desde que não estamos interessados nas variações do funcional gerador com respeito às fontes $\bar{\eta}, \eta$, obtemos então, da eq.(9), para o campo de Dirac (igualando as fontes $\bar{\eta}$ e $\eta$ a zero)

$$
\mathcal{Z}=\left\langle 0_{+} \mid 0_{-}\right\rangle_{\alpha}=\exp \left(i W_{\alpha}\left[A^{e}\right]\right)=N \int_{C_{\alpha}} \mathcal{D} \bar{\psi} \mathcal{D} \psi \exp \left\{-i \int d^{4} x \bar{\psi}(\gamma \cdot \Pi+m-i \epsilon) \psi\right\}
$$

com a convergência da integral sendo garantida pela substituição $m \rightarrow m-i \epsilon, \epsilon>0$. O subscrito $\alpha$ significa que a integração funcional se realiza sobre o espaço de funções que satisfazem as seguintes condições de contorno (c.c)

$$
\begin{aligned}
\psi(r, \theta, z=a) & =\exp (i \alpha) \psi(r, \theta, z=0) \\
\bar{\psi}(r, \theta, z=a) & =\exp (-i \alpha) \bar{\psi}(r, \theta, z=0) .
\end{aligned}
$$

A integral de Fresnel em (10) pode ser determinada de acordo com a relação

$$
\int_{C_{\alpha}} \mathcal{D} \bar{\xi} \mathcal{D} \xi \exp \{-\bar{\xi} \mathrm{K} \xi\}=\operatorname{det} \mathrm{K}
$$

portanto,

$$
\begin{aligned}
\left\langle 0_{+} \mid 0_{-}\right\rangle_{\alpha} & =\exp \left(i W_{\alpha}\left[A^{e}\right]\right)=\operatorname{det}[i(\gamma \cdot \Pi+m)] \\
& \Rightarrow W_{\alpha}\left[A^{e}\right]=-i \ln \operatorname{det}(\gamma \cdot \Pi+m) .
\end{aligned}
$$

Em razão da identidade formal $\operatorname{det}(\exp A)=\exp (\operatorname{Tr} A)$, observamos também que

$$
\text { se } A=\ln B \quad \text { então } \quad \ln \operatorname{det} B=\operatorname{Tr} \ln B,
$$

conseqüentemente, vale para a eq.(14) que

$$
\begin{aligned}
W_{\alpha}\left[A^{e}\right] & =-i \ln \operatorname{det}(\gamma \cdot \Pi+m) \\
& =-i \operatorname{Tr} \ln \left[\mu^{-1}(\gamma \cdot \Pi+m)\right] \\
& =-i \operatorname{Tr} \ln [(\gamma \cdot \Pi+m)] \\
& +i \operatorname{Tr} \ln [(\gamma \cdot P+m)],
\end{aligned}
$$

onde $\mu=(\gamma \cdot P+m)$ surge em razão de uma normalização conveniente na definição do funcional gerador

$$
W_{\alpha}\left[A^{e} \rightarrow 0\right]=0 \Rightarrow Z_{\alpha}\left[A^{e}\right]=\exp \left(i W_{\alpha}\left[A^{e}\right]\right) \rightarrow 1 .
$$

Por outro lado, do método da função zeta generalizada [21] temos a seguinte relação

$$
\operatorname{det} B=\exp \left(-\zeta^{\prime}(s=0)\right) \Rightarrow \ln \operatorname{det} B=-\zeta^{\prime}(s=0)
$$

ou seja, se pudermos calcular a derivada da função zeta associada determinaremos $\ln \operatorname{det} B$, e portanto, o determinante de interesse.

O desenvolvimento do traço na eq.(15) ocorrerá no contexto do formalismo de $2^{a}$ ordem.

\section{A RESOLUÇÃO DO TRAÇO DO OPERADOR EM (15)}

Sabemos que det $(m \pm \gamma \cdot \Pi)$ é um escalar de Lorentz, o que significa que o mesmo não depende do sinal de $\gamma \cdot \Pi$, i.e.,

$$
\operatorname{det}(m+\gamma \cdot \Pi)=\operatorname{det}(m-\gamma \cdot \Pi)
$$

Com isso, temos que [20]

$$
\begin{aligned}
\ln \operatorname{det}(m+\gamma \cdot \Pi)= & \frac{1}{2}(\ln \operatorname{det}(m+\gamma \cdot \Pi) \\
& +\ln \operatorname{det}(m-\gamma \cdot \Pi)) \\
= & \frac{1}{2}(\operatorname{Tr} \ln (m+\gamma \cdot \Pi) \\
& +\operatorname{Tr} \ln (m-\gamma \cdot \Pi)) \\
= & \frac{1}{2} \operatorname{Tr} \ln \left(m^{2}-(\gamma \cdot \Pi)^{2}\right) .
\end{aligned}
$$

Por outro lado, 


$$
-(\gamma \cdot \Pi)^{2}=-\gamma^{\mu} \gamma^{\nu} \Pi_{\mu} \Pi_{\nu}=-\left(\frac{1}{2}\left\{\gamma^{\mu}, \gamma^{\nu}\right\}+\frac{1}{2}\left[\gamma^{\mu}, \gamma^{\nu}\right]\right) \Pi_{\mu} \Pi_{\nu}=-\left(-g^{\mu \nu}-i \sigma^{\mu \nu}\right) \Pi_{\mu} \Pi_{\nu}=\Pi^{2}-i \sigma^{\mu \nu} \Pi_{\mu} \Pi_{\nu}
$$

onde a assinatura da métrica vale +2 . Mas,

$$
\begin{aligned}
\sigma^{\mu \nu} \Pi_{\mu} \Pi_{\nu} & =\frac{1}{2} \sigma^{\mu \nu}\left(\Pi_{\mu} \Pi_{\nu}-\Pi_{\nu} \Pi_{\mu}\right)=\frac{1}{2} \sigma^{\mu \nu}\left(\left(\frac{1}{i} \partial_{\mu}-e A_{\mu}^{e}\right)\left(\frac{1}{i} \partial_{\nu}-e A_{\nu}^{e}\right)-\left(\frac{1}{i} \partial_{\nu}-e A_{\nu}^{e}\right)\left(\frac{1}{i} \partial_{\mu}-e A_{\mu}^{e}\right)\right) \\
& =\frac{1}{2} \sigma^{\mu \nu}\left(-e \frac{1}{i} \partial_{\mu} A_{\nu}^{e}+\frac{1}{i} e \partial_{\nu} A_{\mu}^{e}\right)=-\frac{1}{i} \frac{e}{2} \sigma^{\mu \nu} F_{\mu \nu}
\end{aligned}
$$

onde no caso em questão de campo constante e uniforme resulta

$$
\sigma^{\mu \nu} \Pi_{\mu} \Pi_{\nu}=-\frac{1}{i} \frac{e}{2} \sigma^{\mu \nu} F_{\mu \nu}=\frac{1}{i} e B \sigma^{3} .
$$

Com isto a expressão em (18) torna-se

$$
\frac{1}{2} \operatorname{Tr} \ln \left(m^{2}-(\gamma \cdot \Pi)^{2}\right)=\frac{1}{2} \operatorname{Tr} \ln \left(\left(m^{2}+\Pi^{2}\right) \mathrm{I}_{4}-e B \sigma^{3}\right) .
$$

Vamos calcular o traço na eq.(22). Designando por $\mathrm{AV}_{i}[\mathrm{X}]$, o i-ésimo autovalor do operador $\mathrm{X}$ temos

$$
\begin{aligned}
& \operatorname{Tr} \ln \left(\left(m^{2}+\Pi^{2}\right) \mathrm{I}_{4}-e B \sigma^{3}\right)=\operatorname{tr}_{x} \operatorname{tr}_{\gamma} \ln \left(\left(m^{2}+\Pi^{2}\right) \mathrm{I}_{4}-e B \sigma^{3}\right) \\
& =\operatorname{tr}_{x} \sum_{i=1}^{4} \ln \mathrm{AV}_{i}\left[\begin{array}{cccc}
m^{2}+\Pi^{2}-e B & 0 & 0 & 0 \\
0 & m^{2}+\Pi^{2}+e B & 0 & 0 \\
0 & 0 & m^{2}+\Pi^{2}-e B & 0 \\
0 & 0 & 0 & m^{2}+\Pi^{2}+e B
\end{array}\right] \\
& =2 \operatorname{tr}_{x}\left[\ln \left(m^{2}+\Pi^{2}-e B\right)+\ln \left(m^{2}+\Pi^{2}+e B\right)\right] \text {. }
\end{aligned}
$$

Substituindo o resultado (23) em (22) conseguimos

$$
\begin{aligned}
& \frac{1}{2} \operatorname{Tr} \ln \left(m^{2}-(\gamma \cdot \Pi)^{2}\right) \\
= & \operatorname{tr}_{x}\left[\ln \left(m^{2}+\Pi^{2}-e B\right)+\ln \left(m^{2}+\Pi^{2}+e B\right)\right]
\end{aligned}
$$

e usando (18) e (15) temos como resultado

$$
\begin{aligned}
& -i \operatorname{tr}_{x}\left[\ln \left(m^{2}+\Pi^{2}-e B\right)+\ln \left(m^{2}+\Pi^{2}+e B\right)\right] \\
= & -i\left[\ln \operatorname{det}_{x}\left(m^{2}+\Pi^{2}-e B\right)\right. \\
& \left.+\ln \operatorname{det}_{x}\left(m^{2}+\Pi^{2}+e B\right)\right] .
\end{aligned}
$$

Um desenvolvimento análogo a este permite concluir para a segunda parte do traço em (15)

$$
\begin{aligned}
& \frac{1}{2} \operatorname{Tr} \ln \left(\left(m^{2}+P^{2}\right) \mathrm{I}_{4}\right)=2 \operatorname{tr}_{x} \ln \left(m^{2}+P^{2}\right) \\
= & 2 \ln \operatorname{det}_{x}\left(m^{2}+P^{2}\right) .
\end{aligned}
$$

Reunindo as contribuições (24) e (25) obtemos

$$
\begin{aligned}
W_{\alpha}\left[A^{e}\right] & =-i\left[\ln \operatorname{det}_{x}\left(m^{2}+\Pi^{2}-e B\right)\right. \\
& \left.+\ln \operatorname{det}_{x}\left(m^{2}+\Pi^{2}+e B\right)\right] \\
& +2 i \ln \operatorname{det}_{x}\left(m^{2}+P^{2}\right)
\end{aligned}
$$

Observando (16), a eq.(26) reescreve-se

$$
\begin{aligned}
W_{\alpha}\left[A^{e}\right] & =i \zeta^{\prime}\left(s=0, H_{1}\right)+i \zeta^{\prime}\left(s=0, H_{2}\right) \\
& -2 i \zeta^{\prime}\left(s=0, H_{0}\right)
\end{aligned}
$$

com

$$
\begin{aligned}
& H_{1}=m^{2}-i \epsilon+\Pi^{2}-e B \\
& H_{2}=m^{2}-i \epsilon+\Pi^{2}+e B \\
& H_{0}=m^{2}-i \epsilon+P^{2}
\end{aligned}
$$

\section{A EUCLIDEANIZAÇÃO DA EXPRESSÃO}

A passagem da eq.(27) para o espaço euclideano traz a vantagem de assegurar uma manipulação com operadores cujos autovalores são reais e positivos, condição que permite garantir a existência e definir de forma matematicamente correta funções zeta generalizadas associadas àqueles operadores. Para passar ao espaço euclideano fazemos a mudança $t \rightarrow-i \tau$. Assim,

$$
\left.\exp \left(i W_{\alpha}\left[A^{e}\right]\right) \rightarrow \exp \left(-\mathcal{E}(a) T_{E}\right)\right|_{T_{E} \rightarrow \infty}
$$

de onde segue que

$$
i W_{\alpha}\left[A^{e}\right]=-\mathcal{E}(a) T_{E} \rightarrow \mathcal{E}(a)=-\frac{i}{T_{E}} W_{\alpha}\left[A^{e}\right]
$$

Também, devemos euclideanizar o seguinte operador pre- 
sente nos termos da eq. $(27)$

$$
\begin{aligned}
\Sigma^{2} & :=m^{2}+\Pi^{2}=m^{2}+\left(\frac{1}{i} \partial-e A\right)^{2} \\
& =m^{2}+\left(\frac{1}{i} \partial_{0}-e A_{0}\right)^{2}+\left(\frac{1}{i} \partial_{3}-e A_{3}\right)^{2} \\
& +\sum_{j=1}^{2}\left(\frac{1}{i} \partial_{j}-e A_{j}\right)^{2} \\
& =m^{2}-\partial_{\tau}^{2}-\partial_{3}^{2}+(\vec{p}-e \vec{A})_{\perp}^{2},
\end{aligned}
$$

onde o subscrito $\perp$ significa que estão envolvidas apenas as coordenadas 1 e 2 (coordenadas ortogonais a 3 ) e o potencial, $A_{\mu}^{e}=(0,-B y, 0,0)$, é aquele que gera um campo magnético constante e uniforme, i.e.,

$$
F_{\mu \nu}=\left\{\begin{array}{ccc}
0, & \text { se } & \mu, \nu=0 \\
0, & \text { se } & \mu, \nu=3 \\
B, & \text { se } & \mu=1, \nu=2
\end{array} .\right.
$$

\section{DETERMINAÇÃO DO ESPECTRO DO OPERADOR $\left(m^{2}+\Pi^{2}\right)$}

Da eq.(27) podemos escrever

$$
\zeta_{r e g}(s)=\zeta\left(s, H_{1}\right)+\zeta\left(s, H_{2}\right)-2 \zeta\left(s, H_{0}\right) .
$$

Lembrando da definição da função zeta generalizada

$$
\zeta(s)=\operatorname{Tr} A^{-s},
$$

percebemos que devemos determinar os autovalores do operador de interesse, neste caso de $\left(m^{2}+\Pi^{2}\right)$, i.e., devemos resolver a equação de autovalor a seguir

$$
\left(m^{2}-\partial_{\tau}^{2}-\partial_{3}^{2}+(\vec{p}-e \vec{A})_{\perp}^{2}\right) \chi=\beta \chi
$$

Utilizando o método de separação de variáveis, propomos então que $\chi=R T$, logo

$$
\frac{1}{R}\left(m^{2}-\beta-\partial_{\tau}^{2}-\partial_{3}^{2}+(\vec{p}-e \vec{A})_{\perp}^{2}\right) R=\frac{\partial_{\tau}^{2} T}{T}=-k_{0}^{2}
$$

e desta equação obtemos

$$
\begin{aligned}
\ddot{T}(\tau)+k_{0}^{2} T(\tau) & =0 \\
\left(m^{2}+k_{0}^{2}-\beta-\partial_{3}^{2}+(\vec{p}-e \vec{A})_{\perp}^{2}\right) R(\rho, \varphi, z) & =0 .
\end{aligned}
$$

Na segunda equação acima novamente propomos a separação $R=R_{\perp} Z$, assim

$$
\frac{1}{R_{\perp}}\left(m^{2}+k_{0}^{2}-\beta+(\vec{p}-e \vec{A})_{\perp}^{2}\right) R_{\perp}=\frac{\partial_{3}^{2} Z}{Z}=-k_{3}^{2}
$$

e conseqüentemente

$$
\begin{aligned}
\ddot{Z}(z)+k_{3}^{2} Z(z) & =0 \\
(\vec{p}-e \vec{A})_{\perp}^{2} R_{\perp}(\rho, \varphi)+k_{\perp}^{2} R_{\perp}(\rho, \varphi) & =0
\end{aligned}
$$

onde fizemos $k_{\perp}^{2}=\beta-m^{2}-k_{0}^{2}-k_{3}^{2}$. Sobre a variável $z$ impõe-se a condição de contorno (11),

$$
Z(a)=\exp (i \alpha) Z(0)
$$

A solução geral da eq.(40) tem a seguinte forma

$$
Z(z)=C_{1} \exp \left(i k_{3} z\right)+C_{2} \exp \left(-i k_{3} z\right)
$$

e tendo em conta a condição (42) obtemos

$$
\begin{aligned}
Z(a) & =C_{1} \exp \left(i k_{3} a\right)+C_{2} \exp \left(-i k_{3} a\right) \\
& =\exp (i \alpha)\left(C_{1}+C_{2}\right)=Z(0)
\end{aligned}
$$

o que implica em

$$
\begin{aligned}
& \left(C_{1}+C_{2}\right) \cos \left(k_{3} a\right)+i\left(C_{1}-C_{2}\right) \operatorname{sen}\left(k_{3} a\right) \\
= & \left(C_{1}+C_{2}\right) \cos \alpha+\left(C_{1}+C_{2}\right) \operatorname{sen} \alpha
\end{aligned}
$$

ou ainda

$$
\begin{aligned}
\cos \left(k_{3} a\right)= & \cos \alpha \Rightarrow k_{3}=\frac{\alpha+2 \pi l}{a}, \quad l \in \mathbb{Z} \\
\text { e } \quad C_{2}=0 . &
\end{aligned}
$$

Por outro lado, a solução para a eq.(41) é conhecida, são aos níveis de Landau [22]. Estes níveis constituem a solução da equação de autovalor para o problema do elétron submetido a um campo magnético externo uniforme, descrito pela hamiltoniana

$$
H=\frac{1}{2 m}(\vec{p}-e \vec{A})^{2}
$$

e que são dados de acordo com

$$
E_{n}=\left(n+\frac{1}{2}\right) \omega_{c}, \quad n \in \mathbb{N}
$$

onde $\omega_{c}=\frac{e \hbar B}{m c}$ é a freqüência de cíclotron. Sendo assim, os autovalores em (41) tem a forma (em u.n.)

$$
k_{\perp}^{2}=(2 n+1) e B \quad n \in \mathbb{N}
$$

Os autovalores $\beta$ são dados portanto como segue

$$
\beta_{k_{0}, l, n}=m^{2}+k_{0}^{2}+\left(\frac{\alpha+2 \pi l}{a}\right)^{2}+(2 n+1) e B
$$

\section{CONSTRUÇÃO DA FUNÇÃO ZETA GENERALIZADA $\zeta_{\text {reg }}(s)$}

As funções zeta generalizadas que queremos determinar são aquelas presentes em (35). Observando a definição (36) conseguimos 


$$
\begin{aligned}
\zeta\left(s, H_{1}\right) & =T_{E} \frac{e B A}{2 \pi} \sum_{l=-\infty}^{\infty} \sum_{n=0}^{\infty} \int_{-\infty}^{\infty} \frac{d k_{0}}{2 \pi}\left[m^{2}+k_{0}^{2}+\left(\frac{\alpha+2 \pi l}{a}\right)^{2}+2 n e B\right]^{-s} \\
\zeta\left(s, H_{2}\right) & =T_{E} \frac{e B A}{2 \pi} \sum_{l=-\infty}^{\infty} \sum_{n=0}^{\infty} \int_{-\infty}^{\infty} \frac{d k_{0}}{2 \pi}\left[m^{2}+k_{0}^{2}+\left(\frac{\alpha+2 \pi l}{a}\right)^{2}+2(n+1) e B\right]^{-s} \\
2 \zeta\left(s, H_{0}\right) & =2 T_{E} \frac{A a}{(2 \pi)^{4}} \int_{-\infty}^{\infty} d k_{0} d^{3} k\left[m^{2}+k_{0}^{2}+k_{1}^{2}+k_{2}^{2}+k_{3}^{2}\right]^{-s} .
\end{aligned}
$$

O fator $\frac{e B A}{2 \pi}$ que aparece nas expressões (48) e (49) é o fator de contagem para a degenerescência dos níveis de Landau e surge pelo fato de estarmos considerando o cálculo da densidade de energia do campo fermiônico sob condições de contorno e na presença de um campo externo através do problema auxiliar para uma partícula no mesmo contexto físico, uma vez que a equação do primeiro problema, em uma dada etapa, é precisamente a mesma. A explicação para este fator é dada no apêndice. Em (50) $T_{E} A a /(2 \pi)^{4}$ é um fator de normalização.

\section{DESENVOLVIMENTO DA FUNÇÃO ZETA GENERALIZADA $\zeta_{\text {reg }}(s)$}

Para avançarmos no cálculo das funções zeta generalizadas dadas em (48) a (50) utilizaremos a seguinte identidade integral [23]

$$
A^{-s}=\frac{1}{\Gamma(s)} \int_{-\infty}^{\infty} d \xi \xi^{s-1} \exp (-A \xi)
$$

Este resultado é válido quando $\operatorname{Re}(s)>0$ e $\operatorname{Re}(A)>0$.

Observamos aqui a importância do fato, como chamado a atenção na etapa 3 , de trabalharmos com operadores cujos autovalores associados são reais e positivos, pois estes satisfazem as condições acima mencionadas.

Aplicando a identidade (51) nas equações (48) a obtemos para a eq.(35)

$$
\begin{aligned}
\zeta_{r e g}(s)= & \frac{1}{\Gamma(s)} T_{E} \frac{e B A}{(2 \pi)^{2}} \sum_{l=-\infty}^{\infty} \sum_{n=0}^{\infty} \int_{-\infty}^{\infty} d k_{0} \int_{0}^{\infty} d \xi \xi^{s-1}\left\{\exp \left[-\xi\left(m^{2}+k_{0}^{2}+\left(\frac{\alpha+2 \pi l}{a}\right)^{2}+2 n e B\right)\right]\right. \\
& \left.+\exp \left[-\xi\left(m^{2}+k_{0}^{2}+\left(\frac{\alpha+2 \pi l}{a}\right)^{2}+2(n+1) e B\right)\right]\right\} \\
& -\frac{1}{\Gamma(s)} \frac{2 T_{E} A a}{(2 \pi)^{4}} \int_{-\infty}^{\infty} d k_{0} d^{3} k \int_{0}^{\infty} d \xi \xi^{s-1} \exp \left[-\xi\left(m^{2}+k_{0}^{2}+k_{1}^{2}+k_{2}^{2}+k_{3}^{2}\right)\right]
\end{aligned}
$$

Desenvolvemos a seguir somente o primeiro conjunto de termos em (52). Podemos escrever

$$
\frac{T_{E}}{\Gamma(s)} \frac{e B A}{(2 \pi)^{2}} \sum_{l=-\infty}^{\infty} \int_{-\infty}^{\infty} d k_{0} \int_{0}^{\infty} d \xi \xi^{s-1} \exp \left[-\xi\left(m^{2}+k_{0}^{2}+\left(\frac{\alpha+2 \pi l}{a}\right)^{2}\right)\right] \sum_{n=0}^{\infty}[\exp (-2 n e B \xi)+\exp (-2(n+1) e B \xi)]
$$

Agora, utilizando a expressão para a somatória da série ${ }^{1}$ geométrica, a primeira exponencial em (53) resulta em

$$
\sum_{n=0}^{\infty} \exp (-2 n e B \xi)=\frac{1}{1-\exp (-2 e B \xi)}=\frac{\exp (e B \xi)}{2 \operatorname{senh}(e B \xi)}
$$

enquanto que para a segunda segue que

$$
\begin{aligned}
& \sum_{n=0}^{\infty} \exp (-2(n+1) e B \xi) \\
= & \exp (-2 e B \xi) \sum_{n=0}^{\infty}(\exp (-2 e B \xi))^{n} \\
= & \frac{\exp (-2 e B \xi)}{1-\exp (-2 e B \xi)}=\frac{\exp (-e B \xi)}{2 \operatorname{senh}(e B \xi)},
\end{aligned}
$$

\footnotetext{
1 A somatória da série geométrica é $\sum_{n=0}^{\infty} x^{n}=\frac{1}{1-x}, \quad|x|<1$.
} 
ou seja, agrupando os termos temos

$$
\sum_{n=0}^{\infty}[\exp (-2 n e B \xi)+\exp (-2(n+1) e B \xi)]=\frac{\cosh (e B \xi)}{\operatorname{senh}(e B \xi)}=\operatorname{coth}(e B \xi)
$$

Substituindo (56) na eq.(53) resulta

$$
\frac{1}{\Gamma(s)} T_{E} \frac{e B A}{(2 \pi)^{2}} \sum_{l=-\infty}^{\infty} \int_{-\infty}^{\infty} d k_{0} \int_{0}^{\infty} d \xi \xi^{s-1} \operatorname{coth}(e B \xi) \exp \left[-\xi\left(m^{2}+k_{0}^{2}+\left(\frac{\alpha+2 \pi l}{a}\right)^{2}\right)\right]
$$

Aplicamos agora sobre (57) a fórmula da somatória de Poisson para resolver a soma em $l$

$$
\sum_{l=-\infty}^{\infty} F(l)=\sum_{r=-\infty}^{\infty}\left\{\int_{-\infty}^{\infty} \exp (2 \pi i r x) F(x) d x\right\}
$$

Portanto, segue, em particular, que

$$
\begin{aligned}
& \sum_{l=-\infty}^{\infty} \exp \left[-\xi\left(\frac{\alpha+2 \pi l}{a}\right)^{2}\right] \\
= & \sum_{r=-\infty}^{\infty}\left\{\int_{-\infty}^{\infty} \exp \left[2 \pi i r x-\xi\left(\frac{\alpha+2 \pi x}{a}\right)^{2}\right] d x\right\},
\end{aligned}
$$

que substituída em (57) fornece

$$
\begin{aligned}
& \frac{1}{\Gamma(s)} T_{E} \frac{e B A}{(2 \pi)^{2}} \sum_{r=-\infty}^{\infty} \int_{-\infty}^{\infty} d k_{0} \int_{0}^{\infty} d \xi \xi^{s-1} \operatorname{coth}(e B \xi) \\
& \times \int_{-\infty}^{\infty} d x \exp \left[2 \pi i r x-\xi\left(m^{2}+k_{0}^{2}+\left(\frac{\alpha+2 \pi x}{a}\right)^{2}\right)\right] .
\end{aligned}
$$

É imediato calcular a integral em $k_{0}$ na eq.(59), seu resultado é

$$
\int_{-\infty}^{\infty} d k_{0} \exp \left[-\xi k_{0}^{2}\right]=\sqrt{\frac{\pi}{\xi}}
$$

conseqüentemente,

$$
\begin{aligned}
& \frac{1}{\Gamma(s)} T_{E} \frac{e B A}{(2 \pi)^{2}} \sqrt{\pi} \sum_{r=-\infty}^{\infty} \int_{0}^{\infty} d \xi \xi^{s-\frac{3}{2}} \operatorname{coth}(e B \xi) \\
& \times \int_{-\infty}^{\infty} d x \exp \left[2 \pi i r x-\xi\left(m^{2}+\left(\frac{\alpha+2 \pi x}{a}\right)^{2}\right)\right] .
\end{aligned}
$$

Agora, determinamos a integral

$$
\int_{-\infty}^{\infty} d x \exp \left[2 \pi i r x-\xi\left(\frac{\alpha+2 \pi x}{a}\right)^{2}\right]
$$

Realizando a mudança de variável $y=\frac{\alpha+2 \pi x}{a}$ temos

$$
\frac{a}{2 \pi} \exp (-i r \alpha) \int_{-\infty}^{\infty} d y \exp \left[i r a y-\xi y^{2}\right]\left(\frac{r a}{2}\right)^{2}\left(\frac{1}{\xi}\right) \int_{-\infty}^{\infty} d y \exp \left[-\left(\sqrt{\xi} y-\frac{i r a}{2 \sqrt{\xi}}\right)^{2}\right]
$$

Novamente, designando $\sqrt{\xi} y=z$ segue que

$$
\int_{-\infty}^{\infty} d x \exp \left[2 \pi i r x-\xi\left(\frac{\alpha+2 \pi x}{a}\right)^{2}\right]=\frac{a}{2 \sqrt{\pi \xi}} \exp \left(-i r \alpha-\left(\frac{r a}{2}\right)^{2} \frac{1}{\xi}\right)
$$

Ou seja, a eq.(61), tendo em conta (64), torna-se

$$
\frac{T_{E}}{\Gamma(s)} \frac{e B A a}{2(2 \pi)^{2}} \sum_{r=-\infty}^{\infty} \exp (-i r \alpha) \int_{0}^{\infty} d \xi \xi^{s-2} \operatorname{coth}(e B \xi) \exp \left(-m^{2} \xi-\left(\frac{r a}{2}\right)^{2} \frac{1}{\xi}\right)
$$

Desde que, a função de Langevin, L $(x)$ é definida em termos da função $\operatorname{coth} x$ como [20]

$$
\mathrm{L}(e B \xi)=\operatorname{coth}(e B \xi)-(e B \xi)^{-1},
$$




$$
\begin{aligned}
& \frac{1}{\Gamma(s)} \frac{T_{E} A a}{2(2 \pi)^{2}} \sum_{r=-\infty}^{\infty} \exp (-i r \alpha) \int_{0}^{\infty} d \xi \xi^{s-3} \exp \left(-m^{2} \xi-\left(\frac{r a}{2}\right)^{2} \frac{1}{\xi}\right) \\
& +\frac{1}{\Gamma(s)} T_{E} \frac{e B A a}{2(2 \pi)^{2}} \sum_{r=-\infty}^{\infty} \exp (-i r \alpha) \int_{0}^{\infty} d \xi \xi^{s-2} \mathrm{~L}(e B \xi) \exp \left(-m^{2} \xi-\left(\frac{r a}{2}\right)^{2} \frac{1}{\xi}\right) .
\end{aligned}
$$

Observando a eq.(67) notamos que no primeiro termo o valor $r=0$ fornece

$$
\frac{1}{\Gamma(s)} \frac{T_{E} A a}{2(2 \pi)^{2}} \int_{0}^{\infty} d \xi \xi^{s-3} \exp \left(-m^{2} \xi\right)
$$

expressão que é cancelada pelo último termo em (52), pois este

$$
-\frac{1}{\Gamma(s)} \frac{2 T_{E} A a}{(2 \pi)^{4}} \int_{-\infty}^{\infty} d k_{0} d^{3} k \int_{0}^{\infty} d \xi \xi^{s-1} \exp \left[-\xi\left(m^{2}+k_{0}^{2}+k_{1}^{2}+k_{2}^{2}+k_{3}^{2}\right)\right]
$$

usando o resultado em (58) reescreve-se como

$$
-\frac{1}{\Gamma(s)} \frac{T_{E} A a}{2(2 \pi)^{2}} \int_{0}^{\infty} d \xi \xi^{s-3} \exp \left(-\xi m^{2}\right) .
$$

Com isto, agrupando (67) e (70), a eq.(52) simplifica-se para

$$
\begin{aligned}
\zeta_{\text {reg }}(s)= & \frac{1}{\Gamma(s)} \frac{T_{E} A a}{2(2 \pi)^{2}} \sum_{\substack{r=-\infty \\
r \neq 0}}^{\infty} \exp (-i r \alpha) \int_{0}^{\infty} d \xi \xi^{s-3} \exp \left(-m^{2} \xi-\left(\frac{r a}{2}\right)^{2} \frac{1}{\xi}\right) \\
& +\frac{1}{\Gamma(s)} T_{E} \frac{e B A a}{2(2 \pi)^{2}} \sum_{r=-\infty}^{\infty} \exp (-i r \alpha) \int_{0}^{\infty} d \xi \xi^{s-2} \mathrm{~L}(e B \xi) \exp \left(-m^{2} \xi-\left(\frac{r a}{2}\right)^{2} \frac{1}{\xi}\right) .
\end{aligned}
$$

Tendo em conta a representação integral para a função de Bessel modificada de ordem $\nu$ [23]

$$
K_{\nu}(z)=\frac{1}{2}\left(\frac{z}{2}\right)^{\nu} \int_{0}^{\infty} \eta^{-\nu-1} \exp \left(-\eta-\frac{z^{2}}{4 \eta}\right) d \eta, \quad|\arg z|<\frac{\pi}{2}, \quad \operatorname{Re}\left(z^{2}\right)>0,
$$

e realizando a mudança de variável $\eta=m^{2} \xi$, a integral em $\xi$ no primeiro termo em (71) é reescrita como

$$
\int_{0}^{\infty} d \xi \xi^{s-3} \exp \left(-m^{2} \xi-\left(\frac{r a}{2}\right)^{2} \frac{1}{\xi}\right)=m^{4-2 s} \int_{0}^{\infty} d \eta \eta^{s-3} \exp \left(-\eta-\frac{(m r a)^{2}}{4 \eta}\right) .
$$

Dessa forma, identificando $z=m r a$ e $\nu=2-s$, segue

$$
\int_{0}^{\infty} d \xi \xi^{s-3} \exp \left(-m^{2} \xi-\left(\frac{r a}{2}\right)^{2} \frac{1}{\xi}\right)=2^{3-s}\left(\frac{m}{r a}\right)^{2-s} K_{2-s}(m r a) .
$$

Substituindo (74) em (71) temos

$$
\begin{aligned}
\zeta_{r e g}(s)=\frac{1}{\Gamma(s)} & \frac{T_{E} A a}{(2 \pi)^{2}}\left\{\left(\frac{2 m}{a}\right)^{2-s} \sum_{\substack{r=-\infty \\
r \neq 0}}^{\infty} \frac{1}{r^{2-s}} K_{2-s}(m r a) \exp (-i r \alpha)\right. \\
& \left.+\left(\frac{e B}{2}\right) \sum_{r=-\infty}^{\infty} \exp (-i r \alpha) \int_{0}^{\infty} \frac{d \xi}{\xi^{2-s}} \mathrm{~L}(e B \xi) \exp \left(-m^{2} \xi-\left(\frac{r a}{2}\right)^{2} \frac{1}{\xi}\right)\right\}
\end{aligned}
$$

Por fim, podemos, na segunda integral, realizar a mudança de variável

$$
\xi=\frac{a^{2}}{\sigma} \Rightarrow d \xi=-\left(\frac{a}{\sigma}\right)^{2} d \sigma
$$


conseqüentemente, segue que

$$
\int_{0}^{\infty} \frac{d \xi}{\xi^{2-s}} \mathrm{~L}(e B \xi) \exp \left(-m^{2} \xi-\left(\frac{r a}{2}\right)^{2} \frac{1}{\xi}\right) \rightarrow \frac{1}{a^{2-2 s}} \int_{0}^{\infty} d \sigma \sigma^{s} \mathrm{~L}\left(\frac{e B a^{2}}{\sigma}\right) \exp \left(-\left(\frac{r}{2}\right)^{2} \sigma-\frac{(a m)^{2}}{\sigma}\right)
$$

e assim, a eq.(75) resulta em

$$
\begin{aligned}
\zeta_{\text {reg }}(s)=\frac{1}{\Gamma(s)} & \frac{T_{E} A a}{(2 \pi)^{2}}\left\{\left(\frac{2 m}{a}\right)^{2-s} \sum_{\substack{r=-\infty \\
r \neq 0}}^{\infty} \frac{1}{r^{2-s}} K_{2-s}(m r a) \exp (-i r \alpha)\right. \\
& \left.+\frac{e B}{2 a^{2-2 s}} \sum_{r=-\infty}^{\infty} \exp (-i r \alpha) \int_{0}^{\infty} \frac{d \sigma}{\sigma^{-s}} \mathrm{~L}\left(\frac{e B a^{2}}{\sigma}\right) \exp \left(-\left(\frac{r}{2}\right)^{2} \sigma-\frac{(a m)^{2}}{\sigma}\right)\right\}
\end{aligned}
$$

\section{CÁlCULO DA DERIVADA DE $\zeta_{r e g}(s)$ E A DENSIDADE DE ENERGIA DE CASIMIR}

Para calcular $\zeta_{r e g}^{\prime}(s=0)$ utilizamos um artifício comum na literatura $[1,21]$

$$
\text { Se } \quad \zeta_{\text {reg }}(s)=\frac{F(s)}{\Gamma(s)} \quad \text { então } \quad \zeta_{\text {reg }}^{\prime}(s=0)=F(s=0)
$$

Com isto é imediato determinar que

$$
\begin{aligned}
\zeta_{r e g}^{\prime}(0)=\frac{T_{E} A a}{(2 \pi)^{2}}\left\{\left(\frac{2 m}{a}\right)^{2} \sum_{\substack{r=-\infty \\
r \neq 0}}^{\infty} \frac{1}{r^{2}} K_{2}(m r a) \exp (-i r \alpha)\right. \\
\left.+\left(\frac{e B}{2 a^{2}}\right) \sum_{r=-\infty}^{\infty} \exp (-i r \alpha) \int_{0}^{\infty} d \sigma \mathrm{L}\left(\frac{e B a^{2}}{\sigma}\right) \exp \left(-\left(\frac{r}{2}\right)^{2} \sigma-\frac{(a m)^{2}}{\sigma}\right)\right\}
\end{aligned}
$$

Observando as equações (27) e (32) obtemos

$$
\begin{aligned}
& \mathcal{E}(a, B)=\frac{1}{T_{E}} \zeta_{r e g}^{\prime}(0)=\frac{A a}{(2 \pi)^{2}}\left\{\left(\frac{2 m}{a}\right)^{2} \sum_{\substack{r=-\infty \\
r \neq 0}}^{\infty} \frac{1}{r^{2}} K_{2}(m r a) \exp (-i r \alpha)\right. \\
& \left.\quad+\left(\frac{e B}{2 a^{2}}\right) \sum_{r=-\infty}^{\infty} \exp (-i r \alpha) \int_{0}^{\infty} d \sigma \mathrm{L}\left(\frac{e B a^{2}}{\sigma}\right) \exp \left(-\left(\frac{r}{2}\right)^{2} \sigma-\frac{(a m)^{2}}{\sigma}\right)\right\}
\end{aligned}
$$

ou ainda, dividindo ambos os membros pela área $A$

$$
\frac{\mathcal{E}(a, B)}{A}=\frac{(a m)^{2}}{\pi^{2} a^{3}} \sum_{\substack{r=-\infty \\ r \neq 0}}^{\infty} \frac{1}{r^{2}} K_{2}(a m r) \exp (-i r \alpha)+\frac{e B}{8 \pi^{2} a} \sum_{r=-\infty}^{\infty} \exp (-i r \alpha) \int_{0}^{\infty} d \sigma \mathrm{L}\left(\frac{e B a^{2}}{\sigma}\right) \exp \left(-\frac{r^{2} \sigma}{4}-\frac{(a m)^{2}}{\sigma}\right) .
$$

Vamos analisar a contribuição para a densidade de energia quando $r=0$ no segundo termo

$$
\mathcal{E}_{r=0}(a, B)=\frac{e B A}{8 \pi^{2} a} \int_{0}^{\infty} d \sigma \mathrm{L}\left(\frac{e B a^{2}}{\sigma}\right) \exp \left(-\frac{(a m)^{2}}{\sigma}\right)
$$


Voltando com a mudança de variável em (76) notamos que

$$
\mathcal{E}_{r=0}(a, B)=\frac{e B A a}{8 \pi^{2}} \int_{0}^{\infty} d \xi \mathrm{L}(e B \xi) \exp \left(-m^{2} \xi\right)
$$

ou seja,

$$
\frac{\mathcal{E}_{r=0}(B)}{A a}=\frac{e B}{8 \pi^{2}} \int_{0}^{\infty} d \xi \mathrm{L}(e B \xi) \exp \left(-m^{2} \xi\right) .
$$

Esta parcela contribui como uma densidade de energia uniforme e constante em todo o espaço, e portanto não tem importância para o efeito Casimir. Com isto a eq.(82) reduz-se a

$$
\frac{\mathcal{E}(a, B)}{A}=\frac{(a m)^{2}}{\pi^{2} a^{3}} \sum_{\substack{r=-\infty \\ r \neq 0}}^{\infty} \frac{1}{r^{2}} K_{2}(a m r) \exp (-i r \alpha)+\frac{e B}{8 \pi^{2} a} \sum_{\substack{r=-\infty \\ r \neq 0}}^{\infty} \exp (-i r \alpha) \int_{0}^{\infty} d \sigma \mathrm{L}\left(\frac{e B a^{2}}{\sigma}\right) \exp \left(-\frac{r^{2} \sigma}{4}-\frac{(a m)^{2}}{\sigma}\right) .
$$

\section{ANÁllSE DA EQ.(86) PARA OS VALORES}

$$
\alpha=0 \mathbf{E} \alpha=\pi
$$

O resultado em (86) é o resultado final de nosso cálculo. Ele exibe claramente um termo que independe do campo magnético $B$ e um outro dependente deste. Assim, no primeiro termo existe a influência apenas da condição de contorno, expressa através do parâmetro $\alpha$, sobre a energia de Casimir enquanto que no segundo há uma mistura das duas influências, devida a condição de contorno e devida ao campo magnético externo.
Observamos que o valor para $r=0$ está descartado em ambas as somas em (86) e isto ocorre em razão do processo de renormalização que conduz à energia de Casimir sob consideração. Para extrair informações do resultado geral em (86) vamos considerar a seguir as situações limites do parâmetro $\alpha$ para os valores 0 e $\pi$.

Na situação que $\alpha=0$, a exponencial simplifica-se como segue

$$
\exp (-i r \alpha) \rightarrow 1
$$

neste caso, a eq.(82) assume a seguinte forma

$$
\frac{\mathcal{E}(a, B)}{A}=\frac{2(a m)^{2}}{\pi^{2} a^{3}} \sum_{r=1}^{\infty} \frac{1}{r^{2}} K_{2}(a m r)+\frac{e B}{4 \pi^{2} a} \sum_{r=1}^{\infty} \int_{0}^{\infty} d \sigma \mathrm{L}\left(\frac{e B a^{2}}{\sigma}\right) \exp \left(-\left(\frac{r}{2}\right)^{2} \sigma-\frac{(a m)^{2}}{\sigma}\right) .
$$

Este é o resultado em que a condição de contorno periódica se impõe sobre o campo fermiônico.

De mais interesse aqui será detalhar as conseqüências da situação para $\alpha=\pi$.

Neste caso a exponencial contribui com

$$
\exp (-i r \alpha) \rightarrow(-1)^{r}
$$

e com isto, obtemos nesta situação

$$
\begin{aligned}
\frac{\mathcal{E}(a, B)}{A}= & -\frac{2(a m)^{2}}{\pi^{2} a^{3}} \sum_{r=1}^{\infty} \frac{(-1)^{r-1}}{r^{2}} K_{2}(a m r) \\
& -\frac{e B}{4 \pi^{2} a} \sum_{r=1}^{\infty}(-1)^{r-1} \int_{0}^{\infty} d \sigma \mathrm{L}\left(\frac{e B a^{2}}{\sigma}\right) \exp \left(-\left(\frac{r}{2}\right)^{2} \sigma-\frac{(a m)^{2}}{\sigma}\right) .
\end{aligned}
$$

A eq.(89) mostra concordância com os resultados encontrados nas referências [2, 18], onde são aplicados diferentes métodos de abordagem ao problema, precisamente o de Schwinger, com a representação de tempo próprio, e o de soma de modos.

Notamos que a primeira contribuição em (89) independe do campo magnético e tem como expressão limite no caso em que $m \rightarrow 0$ exatamente (4) com $\xi=7 \times 4$ como é esperado. Por outro lado, a contribuição dependente do campo magnético $B$ é governado por uma quadratura, estritamente positiva, que decresce monotonicamente quando $n$ cresce e tende a zero no limite $n \rightarrow \infty$ [2]. Com isso o critério de Leibniz aplicado à série alternante convergente garante que o campo magnético externo aumenta a energia de Casimir fermiônica.

É interessante obter de (89) sua expressão limite no regime de fortes campos magnéticos. Nessa situação, a integral é governada pela função exponencial com valor máximo 
dado por $\exp (-a m n)$ no ponto $\sigma=2 a m / n$ e, por sua vez, a função de Langevin pode ser substituída por $1-\xi^{-1}$ no regime de campo forte. Temos duas situações limites neste regime dadas por:

$$
\begin{aligned}
& a m \ll 1 \equiv|B| \gg\left|\varphi_{0}\right| / a^{2}, \\
& a m \gg 1 \equiv|B| \gg\left(\left|\varphi_{0}\right| / a^{2}\right)\left(2 \pi a / \lambda_{C}\right),
\end{aligned}
$$

onde $\phi_{0}$ é o fluxo fundamental $1 / e$ e $\lambda_{C} / 2 \pi$ é o comprimento de onda de Compton $1 / m$ (em unidades naturais). Tendo em conta uma das representações integrais da função de Bessel modificada [23] obtemos:

$$
\frac{\mathcal{E}(a, B)}{\ell^{2}}=-\frac{e B m}{\pi^{2}} \sum_{n=1}^{\infty} \frac{(-1)^{n-1}}{n} K_{1}(a m n) .
$$

Do termo principal da série acima e da expansão assintótica da função de Bessel [23] surgem as expressões nos limites de massa muito pequena e muito grande, respectivamente:

$$
\begin{aligned}
& \frac{\mathcal{E}(a, B)}{\ell^{2}}=-\frac{e B}{12 a} \quad(a m \ll 1), \\
& \frac{\mathcal{E}(a, B)}{\ell^{2}}=-\frac{(a m / 2)^{1 / 2} e B}{\pi^{3 / 2} a} e^{-2 a m} \quad(a m \gg 1) .
\end{aligned}
$$

\section{CONCLUSÕES}

Neste trabalho investigamos tanto a influência das condições de contorno quanto a presença de campos externos sobre o efeito Casimir. No caso de campos bosônicos tem sido verificado uma inibição dessa energia quando campos magnéticos externos e uniformes estão presentes, ao passo que quando considera-se campos fermiônicos a energia de Casimir, ao contrário, é ressaltada, tanto quando se considera campos massivos quanto não-massivos.

A investigação da energia de Casimir de um campo fermiônico sob a influência de um campo magnético externo e uniforme e sob as condições de contorno generalizadas na forma (11) e (12) é realizada usando o método da função zeta generalizada, uma vez que este método permite a obtenção de um resultado com um mínimo de termos espúrios, como exemplificado pelo cálculo efetuado neste trabalho.

O resultado geral obtido para a energia de Casimir em (86) é analisado em termos de suas expressões particulares para os valores $\alpha=0$ (condição de contorno periódica) e $\alpha=\pi$ (condição de contorno antiperiódica). Este última corrobora os resultados conhecidos na literatura $[1,2,18]$.
Assim, fica mais uma vez destacado o fato de que sob a presença de um campo magnético externo a energia de Casimir fermiônica é ressaltada. Ademais, também consideramos as expressões limites de (65) tanto para campo fraco quanto forte, e nesse último caso as expressões resultantes quando a massa é muito pequena ou muito grande.

\section{AGRADECIMENTOS}

Os autores agradecem ao Grupo de Efeito Casimir do Instituto de Física da UFRJ, professores Dr. Marcus Venicius, Dr. Carlos Farina e Dr. Alexandre Tort pelos importantes esclarecimentos e valiosas discussões.

\section{APÊNDICE - O FATOR DE CONTAGEM DE DEGENERESCÊNCIA DOS NÍVEIS DE LANDAU} [22]

Desde que consideramos o movimento do elétron limitado a uma caixa bidimensional $A=L_{x} L_{y}$ e que sua função de onda depende de $x$ na forma $\exp \left(\frac{i}{\hbar} p_{x} x\right)$ vale a condição de periodicidade, i.e.,

$$
\exp \left(\frac{i}{\hbar} p_{x} x\right)=\exp \left(\frac{i}{\hbar} p_{x}\left(x+L_{x}\right)\right) \Rightarrow \frac{1}{\hbar} p_{x} L_{x}=2 \pi n,
$$

ou seja,

$$
p_{x}=\frac{2 \pi \hbar n}{L_{x}} \Rightarrow \delta p_{x}=\frac{2 \pi \hbar}{L_{x}}
$$

com $\delta p_{x}$ correspondendo a medida do menor intervalo possível para $p_{x}$. Dessa forma, o número de valores de $p_{x}$ num intervalo $\triangle p_{x}$ é

$$
N=\frac{\triangle p_{x}}{\delta p_{x}}=\frac{L_{x}}{2 \pi \hbar} \triangle p_{x} .
$$

Agora, os valores admissíveis para $p_{x}$ são todos aqueles dentro da área $A$. Desde que, $y_{0}$, o centro da circunferência clássico, relaciona-se com $p_{x}$ de acordo com

$$
y_{0}=-\frac{c p_{x}}{e B}
$$

e que seu valor máximo é $L_{y}$ segue que

$$
N=\frac{L_{x}}{2 \pi \hbar} \triangle p_{x}=\frac{L_{x}}{2 \pi \hbar} \frac{e B L_{y}}{c}=\frac{e B A}{2 \pi \hbar c} \rightarrow \frac{e B A}{2 \pi} . \quad(\text { em u.n. })
$$

[1] C. Farina, A.C. Tort, M.V. Cougo-Pinto; Fermionic Casimir Effect in an External Magnetic Field In: Workshop on Quantum Field Theory under the Influence of External Conditions, 1998, Leipzig. The Casimir Effect 50 Years Later, Cingapura: World Scientific, 235-239 (1999).

[2] M.V. Cougo-Pinto, C. Farina, A.C. Tort; The Influence of an External Magnetic Field on the Fermionic Casimir Effect, Brazilian J. Phys., 31 (1) (2001) 84.

[3] E. Elizalde, S.D. Odintsov, A. Romeo, A.A. Bytsenko, S.
Zerbini; Zeta Regularization Techniques with Applications, World Scientific (1994).

[4] H. B. G. Casimir, Proc. K. Akad. Wet., B 51793 (1948).

[5] M.S.R. Miltão; Estudo do Efeito Casimir Eletromagnético Esférico pelo Método da Dupla Regularização, Tese de Doutorado, apresentada ao Instituto de Física da Universidade Federal do Rio de Janeiro, RJ. (Não-publicada) (2004).

[6] N. G. van Kampen, B. R. A. Nijboer and K. Schram; On 
the Macroscopic Theory of Van der Waals Forces, Phys. Lett. A 26307 (1968).

[7] J. Schwinger, L. L. DeRaad Jr. and K. A.Milton; Casimir Effect in dielectrics Ann. Phys. (N.Y.) 115, 1 (1978).

[8] B. S. Dewitt; Quantum Field Theory in Curved Spacetime Phys. Rep. 19295 (1975).

[9] S. Nam; Casimir Force in Compact Noncommutative Extra Dimensions and Radius Stabilization, J. High Energy Phys. 10044 (2000). M. Chaichian, A. Demichev, P. Prešnajder, M.M. Sheik-Jabbari and A. Tureanu; Quantum Theories on Noncommutative Spaces with Nontrivial Topology: Aharonov-Bohm and Casimir Effects, Nucl. Phys. B 611383 (2001). W. H. Huang; Casimir Effect on the Radius Stabilization of the Noncommutative Torus, Phys. Lett. B 497317 (2001).

[10] J. F. M. Mendes; Efeitos de vácuo em teoria quântica de campos, Tese de Doutorado, apresentada ao Instituto de Física da Universidade Federal do Rio de Janeiro, RJ. (Não-publicada) (2003). J. Lukierski, A. Nowicki and H. Ruegg, New Quantum Poincaré Algebra and $\kappa$-Deformed Field Theory, Phys. Lett. B 293 (3-4) 344 (1992). M. V. Cougo-Pinto, C. Farina and J. F.M.Mendes, Casimir Effect and Creation of Radiation in Confined $\kappa$-Deformed Electrodynamics, Phys. Lett. B 529 (3-4) 256 (2002).

[11] P. Kosinski, J. Lukierski and P. Maslanka, Local $D=4$ Field Theory on $\kappa$-Deformed Minkowski Space Phys. Rev. D 62025004 (2000).

[12] M. J. Sparnaay, Measurements of Attractive Forces between Flat Plates, Physica 24751 (1958).

[13] S. K. Lamoreaux, Demonstration of the Casimir Force in the 0.6 to $6 \mu \mathrm{m}$ Range, Phys. Lett. 785 (1997).

[14] U. Mohideen and A. Roy; Precision Measurement of the Casimir Force from 0.1 to $0.9 \mu \mathrm{m}$, Phys. Rev. Lett. 81
4549 (1998). A. Roy and U. Mohideen; Demonstration of the Nontrivial Boundary Dependence of the Casimir Force, Phys. Rev. Lett. 824380 (1999).

[15] K. Johnson; The M.I.T. Bag Model, Acta Phys. Polonica B 6865 (1975).

[16] K. Milton; Zero Point Energy of Confined Fermions, Phys. Rev. D 22 (6) 1444 (1980). K. Milton; Toward Finite Zero Point Energies in the Bag Model, Phys. Rev. D 27 (2) 439 (1983).

[17] E. Elizalde, M. Bordag, K. Kirsten; Casimir Energy for a Massive Fermionic Quantum Field with a Spherical Boundary, J. Phys. A 311734 (1998).

[18] D.H. Correa, E.M. Santangelo; Energías de Vacio para el Problema de Landau Relativista, Trabajo de Diploma, UNLP. arXiv: hep-th/0008223 (2000).

[19] M.V. Cougo-Pinto, C. Farina, M.R. Negrão, A.C. Tort; Bosonic Casimir effect in external magnetic field, J. Physics A: Math. Gen. 32 (24) 4457 (1999).

[20] W. Dittrich, M. Reuter; Effective Lagrangians in Quantum Electrodynamics, Lectures Notes in Physics, vol. 220, Springer-Verlag (1985).

[21] A.T. Leite; Método da Função Zeta Generalizada e Aplicações, Dissertação de Mestrado, apresentada ao Intituto de Física da Universidade Federal do Rio de Janeiro, RJ. (Não-publicada) (1997).

[22] L.D. Landau, E. Lifshitz; Mecânica Quântica (Teoria NãoRelativísta), vol. 2, Mir (1985).

[23] I.S. Gradshteyn, M. Ryzhik; Table of Integrals and Series, Pergamon Press (2001).

[24] C. Farina; Notas de Aula, Curso de Tópicos de Física Teórica, IF-UFRJ (2000). 